\title{
Die Dritte und die Vierte Gewalt in Raumplanungsprozessen
}

\section{Zum wechselseitigen Verhältnis von Medien, Justiz und Politik}

\author{
Christian Diller ${ }^{1}$ \\ Eingegangen: 19. Februar 2017 / Angenommen: 4. Januar 2018 / Online publiziert: 16. Januar 2018 \\ (c) Springer-Verlag GmbH Deutschland, ein Teil von Springer Nature 2018
}

\section{Zusammenfassung}

Auch wenn institutionalisierte Medien und rechtsprechende Justiz zu den Akteuren der Raumplanung gezählt werden können, so ist dennoch in der Raumplanungsforschung sowohl national als auch international nur wenig darüber bekannt, welche Rolle sie in Raumplanungsprozessen im Vergleich zu Akteuren aus Politik, Verwaltung, Zivilgesellschaft und Wirtschaft spielen. Ob und inwieweit beeinflussen sie konkrete Planungsentscheidungen? In anderen Politikfeldern (z. B. der Außen- und Finanzpolitik) ist dagegen vor allem der Einfluss der Medien auf politische Prozesse deutlich besser erforscht und zur Rolle der Justiz gibt es immerhin einige Befunde. Aus diesen Erkenntnissen werden in dem ausschließlich literaturbasierten Beitrag erste Hypothesen für das Politikfeld Raumplanung formuliert. Demnach variieren die Rollen der Medien und der Justiz in den einzelnen Phasen des Politikprozesses (policy cycle) derart, dass sie nahezu komplementär zueinander sind: Während die Rolle der Medien vor allem am Anfang bei der Problemformulierung und dem Agenda-Setting sehr bedeutend sein kann, um dann im weiteren politischen Prozess eher abzunehmen, hat die Justiz in Form von Rechtsprechung eher in den späteren Phasen des Politikprozesses sehr selektiven, dann aber unter Umständen maßgeblichen Einfluss. Gemeinsam können die Akteurgruppen Medien und Justiz Planungsprozesse maßgeblich prägen, daher hat auch ihre gemeinsame Betrachtung in empirischen Untersuchungen Sinn.

Schlüsselwörter Medien · Justiz $\cdot$ Planungsprozesse $\cdot$ Policy cycle

Prof. Dr. Christian Diller

christian.diller@geogr.uni-giessen.de

1 Institut für Geographie, Professur für Raumplanung und Stadtgeographie, Justus-Liebig-Universität Gießen, Senckenbergstraße 1, 35390 Gießen, Deutschland 


\title{
The Third and the Fourth Power in Spatial Planning Processes
}

The Mutual Relationship Between Media, Jurisdiction and Politics

\begin{abstract}
Although institutionalized media and jurisdiction could be counted to the actors of spatial planning, only few is known in international and national spatial planning research about their role in spatial planning processes in relation to actors in politics, administration, civil society and economy. If and to which extend do they have an influence on planning processes? In other policy areas (e.g. foreign and finance policy) - especially the influence of the media on political processe - is quite better explored and for the role of jurisdiction there are at least some findings. Based on this knowledge in this exclusive literature-based article first hypothesises for the policy area spatial planning are formulated. Thus, the roles of media und jurisdiction are varying between the specific phases of the policy cycle in a complementing manner: While the media play an important role in the phases of problem formulation and agenda setting and loose importance in the following political process, the jurisdiction has a selective but probabely leading influence in the later phases. Together, both groups of actors in media and jurisdiction can form planning processes significantly, therefore their common analysis in empirical studies makes sense.
\end{abstract}

Keywords Media $\cdot$ Jurisdiction $\cdot$ Planning processes $\cdot$ Policy cycle

\section{Einleitung}

Die Debatten in der deutschen wie in der internationalen Raumplanungsforschung sind seit Jahren durch die Forderung nach der verstärkten Einbeziehung von Akteuren in Planungsprozesse geprägt. Dieser „Communicative Turn“ (unter anderem Healey 1996; Fischler 2000; Selle 2005) scheint längst zum dominierenden Paradigma der Raumplanungsforschung avanciert zu sein. Dabei stehen meist die - bisweilen nur diffus gefassten - Akteurgruppen „Bürger/ Zivilgesellschaft“ und „Wirtschaft" und ihr Verhältnis zu Politik und Verwaltung im Vordergrund der Debatte. Zwei andere Akteurgruppen bleiben dagegen außerhalb der wissenschaftlichen Betrachtung: zum einen die Medien, zum anderen die Justiz, insbesondere die Rechtsprechung. Beobachtungen in und aus der Praxis ${ }^{1}$ liefern jedoch erste Hinweise, dass der Einfluss dieser beiden Akteurgruppen auf Raumplanungsprozesse zunimmt. In diesem literaturbasierten Beitrag wird daher der Frage nachgegangen, welchen Einfluss Medien und Rechtsprechung auf Raumplanungsprozesse und politische Entscheidungen in anderen Politikfeldern haben und zwar sowohl einzeln als auch gemeinsam sowie in Wechselwirkung zueinander. Welche Rolle spielen sie in Raumplanungsprozessen, über welche Macht verfügen sie durch welche Instrumente, inwieweit sind letztlich Planungsentscheidungen durch diese beiden Akteurgruppen geprägt?

Die Darstellung des Forschungsstandes für das Politikfeld Raumplanung in Kapitel 2 macht deutlich, dass es in

\footnotetext{
1 Priebs, A. (2015): The contribution of governance research for planning theory and practice. Vortrag im Rahmen des Symposiums der Leibniz Universität Hannover zum 75. Geburtstag von Dietrich Fürst am 20. März 2015.
}

Deutschland keine und auch international im Grunde nur eine Untersuchung gibt, die den Einfluss der Medien auf Planungsprozesse systematisch analysiert. Vor diesem Hintergrund wird in Kapitel 3 der internationale Forschungsstand zum Einfluss von Medien und Justiz auf politische Entscheidungen für andere Politikfelder skizziert. Diese Analyse ist insofern besonders ergiebig, als aus ihr einige Hypothesen abgeleitet werden, die auch für das Politikfeld Raumplanung relevant sind. Diese werden abschließend in Kapitel 4 ausgeführt.

\section{Die Rolle von traditionellen Medien und Justiz in Raumplanungsprozessen: ein kaum erforschtes Thema}

Auch wenn in den letzten Jahren der Einsatz neuer sozialer Medien ${ }^{2}$ - und hierbei vor allem ihre Rolle bei der Partizipation in Planungsprozessen - erhebliche Aufmerksamkeit auf sich gezogen hat, so geht es hier dennoch um die traditionellen Medien. Darunter werden die institutionalisierten öffentlichen wie privaten Massenmedien Presse, Funk und Fernsehen mit ihren in der Regel von professionellen Journalisten produzierten Inhalten verstanden

\footnotetext{
${ }^{2}$ Neue Medien wie Internet-Blogs haben zu einer sprunghaften Steigerung von Informations-, Artikulations- und Koordinationsmöglichkeiten geführt. Sie ermöglichen eine mediale Hybridisierung und Konvergenz der Inhalte. Medien können jetzt besser miteinander kombiniert werden und sie werden auch eher kombinierend als substituierend genutzt (Dohle/Bernhard 2014). Ob und wieweit die neuen Medien aber tatsächlich die politischen Entscheidungsprozesse beeinflussen und ob sie, wie noch vor einigen Jahren vermutet (Baringhorst 2009: 610), die Möglichkeit der Politik zur Instrumentalisierung von Medien verringern, bleibt abzuwarten (In der Smitten 2009: $263 \mathrm{ff}$.).
} 
(Schulz 2009: 108; Reinemann 2010: 279). Medien sind demnach selbstständige Organisationen, die nach eigenen Regeln und Zielen handeln (Maurer 2008; Pfetsch/Adam 2008: 16); sie gehören grundsätzlich zu den relevanten Akteuren der Planung (Fürst 2010: 18). Demokratietheoretisch sind sie als ,Vierte Gewalt“ als Teil der Öffentlichen Meinung legitimiert (Wagner 2007). Sie üben eine gewisse Kontrolle über Politik und Administration aus und sind insofern strategiefähige Akteure, als sie dabei ihre Handlungsmacht als Ressource einsetzen. Die alltäglichen Raumplanungsthemen stehen für die Medien allerdings eher im Windschatten ihrer Berichterstattung. Gründe hierfür sind die Komplexität der Sachverhalte und die selbstgewählte, nur fachspezifische Kommunikation der Raumplanung. Gleichwohl haben punktuell in den letzten Jahren einige Planungsverfahren große mediale Resonanz erfahren, wie beispielsweise die Live-Berichterstattung des Mediationsverfahrens Stuttgart 21 im Phoenix-TV 2010 (Brettschneider/Schuster 2013; Maier 2013; Stuckenbrock 2013), oder die häufige Darstellung des pannenreichen Projekts Flughafen BerlinBrandenburg, das sich zum medialen Symbol struktureller Politikdefizite entwickelt hat.

Vor diesem Hintergrund erstaunt es, dass die Frage des Einflusses der Medien auf Raumplanungsprozesse in Deutschland, aber auch international, nahezu unerforscht ist.

\subsection{Die internationale Debatte}

In der internationalen Diskussion finden sich Überlegungen, wie Medien besser für die Kommunikation in Planungsprozessen genutzt werden können. So arbeiteten Johnson und Graves (2011) heraus, dass Reality-TV-Shows erhebliche Potenziale bieten, bei einem breiten Publikum Sensibilität für Raumplanungsthemen wie ÖPNV, Innenstadterneuerung, Suburbanisierung und kommunale Identität $\mathrm{zu}$ wecken, was aus fachlicher Sicht auch durchaus wünschenswert ist.

Es liegt jedoch nur eine einzige ausführlichere Untersuchung vor, in der die Rolle der Medien in Raumplanungsprozessen systematisch, vor allem durch Medieninhaltsanalysen und Expertengespräche, behandelt wird. Edey, Seasons und Whitelaw (2006: 147), die selbst auf dieses Forschungsdefizit hinweisen, untersuchten die Rolle der Medien in den Debatten um den „Oak Ridges Moraine“, einer 1900 Quadratkilometer großen geologischen Landform im zentralen Süden der kanadischen Provinz Ontario. Dort entwickelte sich in den 1990er-Jahren zunehmend ein Konflikt zwischen der Siedlungsentwicklung und den Belangen des Natur- und Landschaftsschutzes. In der Untersuchung wird die Rolle der Medien der sich in den 2000er-Jahren verschärfenden Debatten, die letztlich zu restriktiveren Gesetzen für das Gebiet führten, anhand des ,Issue Atten- tion Cycle“"3 genauer betrachtet. Interessant ist besonders, dass das Thema zwar schon länger virulent war, es aber erst durch einen politischen Fehltritt eines zuständigen Ministers, dem unangemessenes Verhalten bei der Erteilung von Planungsgenehmigungen vorgeworfen wurde, mediale Aufmerksamkeit erhielt (Edey/Seasons/Whitelwa 2006: $148 \mathrm{f}$.). Durch diesen medial attraktiven Skandal gelangte das Thema auf die öffentliche Agenda. Es behielt dort lange Jahre seinen Platz und die Berichterstattung in der untersuchten regionalen Zeitung bezog überwiegend Positionen für die Belange des Naturschutzes und gegen die Siedlungsentwicklung. Die Untersuchung macht deutlich, dass es den lokalen Gruppen, die sich für die Naturschutzbelange einsetzten, zunehmend gelang, Einfluss auf die Medienberichterstattung zu nehmen. Der zunehmende Druck von weiten Teilen der Öffentlichkeit und der Medien trug nach Einschätzung der Autoren dieses Beitrags letztlich zu einer Verschärfung der Naturschutzregelungen bei.

Zur Frage der Rolle der Rechtsprechung auf Planungsentscheidungen liegen international zumindest keine vergleichenden Untersuchungen vor. Die Übertragbarkeit solcher Untersuchungen auf die deutsche Situation wäre allerdings wegen der großen Unterschiede in den Rechtssystemen ohnehin erheblich eingeschränkt. In den USA beispielsweise sind im Vergleich zu Deutschland Planungsgesetze und Pläne mit wesentlich größeren Spielräumen für die rechtliche Abwägung formuliert. Beginnend mit der lokalen Ebene spielen die Gerichte bei der Entscheidung über Verstöße gegen Planungsregelungen eine größere Rolle als in Deutschland (Sullivan 2015: 154). Die Situation in den USA ist daher weniger für den aktuellen Vergleich mit Deutschland geeignet. Sie gibt aber möglicherweise Hinweise darauf, in welche Richtung sich die Situation in Deutschland entwickeln könnte, wenn die Bedeutung der Rechtsprechung größer würde. Untersuchungen zur Frage der Rolle der Rechtsprechung in Planungsprozessen aus europäischen Ländern, deren Rechtssystem mit dem deutschen vergleichbar sind, liegen nicht vor.

\subsection{Die Diskussion in Deutschland}

\subsubsection{Die Rolle der Medien}

In Untersuchungen zu Planungsprozessen in Deutschland werden zwar Medienberichterstattungen herangezogen, die Rolle der Medien wird in den Arbeiten jedoch kaum tiefgehend thematisiert. Dies erstaunt besonders bei Planungspro-

\footnotetext{
${ }^{3}$ Nach diesem von Downs (1972) formulierten Ansatz erhält ein Thema zunächst eine steigende öffentliche Aufmerksamkeit, die dann in der Phase der Diskussionen um die Kosten der Problemlösung ihren Höhepunkt erreicht. Während der Umsetzung der eigentlichen Problemlösung nimmt das öffentliche Interesse dagegen wieder deutlich ab.
} 
zessen, die selbst von hoher medialer Bedeutung waren, wie zum Beispiel „Stuttgart 21“. Hier waren die Auseinandersetzungen kontinuierlich von einer breiten Berichterstattung in lokalen, regionalen, nationalen und zum Teil internationalen Medien begleitet. Dennoch nimmt die Medienberichterstattung selbst in ausführlicheren wissenschaftlichen Analysen zu diesem Thema (Eibl 2012) nur eine untergeordnete Rolle ein. Selbst in dem von Brettschneider und Schuster (2013) herausgegebenen umfassenden Sammelband, in dem es um die Planungsgeschichte, das Schlichtungsverfahren und die Auswirkungen von ,Stuttgart 21“ auf die Landtagswahlen in Baden-Württemberg geht, setzt sich nur einer der insgesamt 15 ausführlichen Beiträge zumindest ansatzweise mit den Medien auseinander: Baumgarten und Rucht (2013) ermittelten in einer Befragung die Sozial- und Einstellungsstruktur der Gegner von ,Stuttgart 21“. Sie kommen zu dem Ergebnis, dass das mediale Bild der Gegner nur zum Teil mit der empirisch durch Befragungen ermittelten Bevölkerungsstruktur übereinstimmt. Der Begriff ,Wutbürger“ kann daher als Überzeichnung und als ,mediales Leuchtfeuer“ (Brettschneider/Schuster 2013: 9) bezeichnet werden. Bemerkenswert ist allerdings, dass in der Untersuchung das angeblich medial gezeichnete Bild als Hypothese unterstellt wird. Diese wird jedoch nicht durch eigene differenzierte Analysen der Medienberichterstattung verifiziert, insofern wurde sie unter Umständen von den Forschern selbst überzeichnet ist.

Immerhin zum Teil wird diese Lücke - bemerkenswerterweise in einer Bachelorarbeit - von Aleksandrov (2015) bearbeitet. Er hat die Berichterstattung über „Stuttgart 21“ von vier überregionalen Zeitungen mittels einer auch quantifizierenden Inhaltsanalyse von 869 Artikeln über einen Zeitraum von einem halben Jahr miteinander verglichen. Seine Ergebnisse sind eindeutig: Die Berichterstattung der vier Zeitungen unterscheidet sich gemäß ihrer jeweiligen politischen Grundlinie. Je linksliberaler die Zeitung ist, desto negativer steht sie dem Projekt „Stuttgart 21“ gegenüber und desto positiver wird über die Gegner des Projekts berichtet. Zudem werden Partizipationselemente gefordert. Wenngleich diese Arbeit wertvolle Aufschlüsse über die tendenziöse Medienberichterstattung liefert, wird jedoch nicht analysiert, ob und wenn ja welchen Einfluss diese Medien auf die politischen Entscheidungen in dem Planungskonflikt im Einzelnen hatten.

Auch in der umfassenden Analyse von Becker-Ritterspach (2015), die die Planungsprozesse an den beiden Flughafenstandorten Frankfurt Rhein-Main und BerlinBrandenburg detailliert betrachtet, stellen institutionalisierte Medien keinen Untersuchungsgegenstand dar. Zwar werden Medienberichte punktuell als Quelle herangezogen, jedoch nicht ausführlich analysiert. Der Einfluss der Medienberichterstattung auf das Planungsverfahren wird nicht ansatzweise thematisiert.
Angesichts der Tatsache, dass selbst in Planungsverfahren mit solch großer medialer Präsenz die Rolle der institutionalisierten Medien in den wissenschaftlichen Analysen praktisch nicht beleuchtet wird, verwundert es nicht, dass das Thema der Medienberichterstattung für den „Alltagsfall der Raumplanung“ (z. B. Bauleitplanung, Sanierungsverfahren, Regionalplanung) ebenfalls praktisch unerforscht ist, obwohl auch hier vor allem die Printmedien auf der lokalen und regionalen Ebene bisweilen eine gewisse Bedeutung haben dürften (Richter 2011: 170).

\subsubsection{Die Rolle der Rechtsprechung}

Was die Rechtsprechung angeht, so gibt es insbesondere bei Infrastrukturgroßprojekten wie etwa Flughäfen, aber auch bei Aufgaben aus der Regionalplanung, z. B. Windenergieanlagen, in Deutschland deutliche Anzeichen einer ,Amerikanisierung' insofern, als Planungsverfahren häufig in Klage- und Gerichtsverfahren münden, in denen dann zum Teil jahrelange Raumplanungsprozesse revidiert werden, was wiederum meist Auswirkungen auf vergleichbare zukünftige Planungsverfahren hat. Ein Grund für diese Entwicklung ist die Zersplitterung des Planungsrechts, woraus gefolgert wird, dass Gerichte ihren Prüfauftrag nicht nur formal, sondern auch materiell verstehen sollten (Kupfer 2014). Die Rechtsprechung der Verwaltungsgerichte hat sich daher in weiten Teilen vom Grundgedanken des Rechtsschutzes entfernt und in Richtung der Sachgerechtigkeit einer gerichtlichen Überprüfung entwickelt (Mehde 2010: 398). Im Rahmen der Bauleitplanung umfasst die gerichtliche Prüfung auch die Abwägung (Keilbach 2009: 45; Merkel 2012: $101 \mathrm{ff}$.). Inwieweit mit dem EAG $\mathrm{Bau}^{4}$ (\$§ 2 III und 214 BauGB) Mitte der 2000er-Jahre die Trennung zwischen der Bewertung des Abwägungsvorganges und des Abwägungsergebnisses diffuser wurde - materielle Abwägungsfehler sind Verfahrensfehler - und damit ein Systemwechsel in Deutschland eingeleitet wurde, ist unter den Fachleuten umstritten (Merkel 2012: 236ff.).

Es liegen jedoch kaum Forschungsergebnisse vor, in denen diese Rolle der Rechtsprechung in deutschen Planungsprozessen genauer betrachtet wird. Hervorzuheben ist lediglich die Untersuchung von Becker-Ritterspach (2015), die vergleichend für zwei Planungsverfahren von Flughäfen die Entscheidungen der Gerichte chronologisch nachzeichnet. Das Verfahren um den Ausbau des Flughafens Frankfurt/ Rhein-Main ist ein Beispiel, wie die Justiz durch Rechtsprechung erst relativ spät den Planungsprozess beeinflusste. Die Entscheidungen der Gerichte wurden von den einzel-

\footnotetext{
${ }^{4}$ Das Europarechtsanpassungsgesetz Bau (EAG Bau), im Langtitel „Gesetz zur Anpassung des Baugesetzbuchs an EU-Richtlinien“, vom 24. Juni 2004 hat als Artikelgesetz einige baurechtlich relevante Gesetze wie das Baugesetzbuch geändert.
} 
nen Konfliktparteien durchaus auch politisch bewertet und es erfolgte eine Revisionsklage (Becker-Ritterspach 2015: 73 ff.). Im Falle des Flughafens Berlin-Brandenburg wurde deutlich, dass es sich bei der Justiz um keinen einheitlichen Akteur handelt, sondern die einzelnen Instanzen zu unterschiedlichen Prüfergebnissen kommen können. Die Urteile zeigen, dass die Gerichte hier durchaus auch materielle raumordnerische Abwägungen und damit Interpretationen vornahmen. So wurde beispielsweise bemängelt, dass das raumordnerische Leitbild der dezentralen Konzentration zu wenig Beachtung gefunden habe (Becker-Ritterspach 2015: $247 \mathrm{ff}$.).

\subsubsection{Zwischenfazit}

Kaum erforscht ist schließlich auch die Frage, ob und wie sich die Akteure ,Medien“ und ,Rechtsprechung' in Planungsverfahren wechselseitig beeinflussen und damit die Politik mitbestimmen. Das Planungsrecht unterscheidet zwischen der unmittelbaren Bürgerbeteiligung und der durch Medien hergestellten Öffentlichkeitsbeteiligung, die in ihrer grundsätzlichen Unabgeschlossenheit und Resonanzfunktion rechtlich anders $\mathrm{zu}$ behandeln ist (Richter 2011: 171).

Als Zwischenfazit kann festgehalten werden, dass für das Politikfeld Raumplanung in Deutschland, aber auch international, ein großer Forschungsbedarf zu den Rollen von institutionalisierten Medien und Rechtsprechung besteht. In anderen Politikfeldern, auf die sich auch die theoretischen Überlegungen aus Politik- und Medienwissenschaften $\mathrm{zu}$ diesem Thema beziehen, ist der Forschungsstand dagegen weiter vorangeschritten (vor allem die US-Außenpolitik, aber auch die US-Haushaltspolitik). Für das Politikfeld Raumplanung ist es daher hilfreich, Erkenntnisse aus anderen Politikfeldern auszuwerten, um daraus Hypothesen zu abzuleiten. Nachfolgend wird dies ausführlicher behandelt.

\section{Die Rollen von Medien und Justiz in anderen Politikfeldern}

\subsection{Die Rolle der Medien}

\subsubsection{Die Medialisierungsthese}

Medieneinflüsse auf die Politik werden schon seit jeher als selbstverständlich unterstellt (Schulz 2009: 103). In den USA dominierte in den 1940er- und 1970er- und dann wieder seit den 1990er-Jahren die These vom starken Medieneinfluss. In den 1950er-, 1960er- und 1980er-Jahren wurde dagegen der Politik eine stärkere Fähigkeit zur Beherrschung der Medien zugesprochen (Schulz 2009: 106;
Classen/Arnold 2010: 13; Mergel 2010: 32). Mit der Medialisierungsthese wird behauptet, dass in allen modernen Gesellschaften die Kommunikation zwischen den Subsystemen wie Politik, Gesellschaft und Wirtschaft zunehmend von Medien durchdrungen werde. Je mehr die Politik selbst hektischer, komplexer und umstrittener geworden auf Publizität angewiesen ist, desto stärker muss sie ihre Handlungslogik den Medien anpassen (Vowe/Dohle 2008: 11, 14, 16). Für den ersten Beleg dieser Medialisierungsthese dienen häufig spektakuläre Beispiele von Politikerkarrieren, die durch Medien beendet wurden. Nicht politische Fehler selbst oder deren Aufklärung führen zu Rücktritten, sondern der unprofessionelle mediale Umgang mit den Befunden der Aufklärung. Zweifellos hat die mediale Logik Einfluss auf die Gestaltung des Politikprozesses (Vowe/Dohle 2007: 344), was etwa die Themenselektion, die verkürzten Reaktionszeiten, den Zwang zu vereinfachender Zuspitzung, den Trend zum „Infotainment“ (Bernhard 2012) sowie die Privatisierung der Politikersphäre angeht (Classen/Arnold 2010: 16). Vor allem die Frage des Einflusses der Medien auf Wahlen ist relativ intensiv erforscht (Walthert 2013: 11). Unter welchen Bedingungen aber welche Medienwirkungen auf die Politik mit welchen Folgen eintreten und inwieweit Medien konkrete politische Entscheidungen auch inhaltlich beeinflussen, ist für die Kommunikationswissenschaften eine nicht abschließend geklärte Frage (Richter 2011: 169; Vowe/Dohle 2008: 24). Der empirische Forschungsstand hierzu hat sich in den letzten Jahren jedoch verbessert (Reinemann 2010: 279). Beispielsweise kommt eine Metaanalyse von 19 Policy-Agenda-Studien zum Ergebnis, dass zwölf Studien einen starken, drei Studien einen schwachen und vier Studien keinen Medieneinfluss auf die Politik nachweisen (Walgrave/Van Aelst 2006: 90; Fawzi 2014a: 446).

Die Medien- und Politikwissenschaften haben auf diesen Grundlagen diverse Modelle entwickelt, um die Rolle der Medien im Politikgestaltungsprozess zu fassen (Maurer 2013: 16f.). Einige dieser Modelle beziehen sich auf den Politikprozess (,Policy-Cycle“) (Jann/Wegrich 2014: 106; Jarren/Donges 2011: 205; Lauth/Thiery 2012: 276). In diesem wird davon ausgegangen, dass gesellschaftlich definierte Probleme im System von Politik und Verwaltung stufenweise - die Stufen sind Problemdefinition und Agenda-Setting, Politikformulierung, Implementation, Evaluierung, Terminierung - bearbeitet und gelöst oder zumindest neu definiert werden. Die Phasengliederung des Policy-Cycle liegt auch dem in Abbildung 1 dargestellten Ansatz für empirische Untersuchungen zugrunde. 


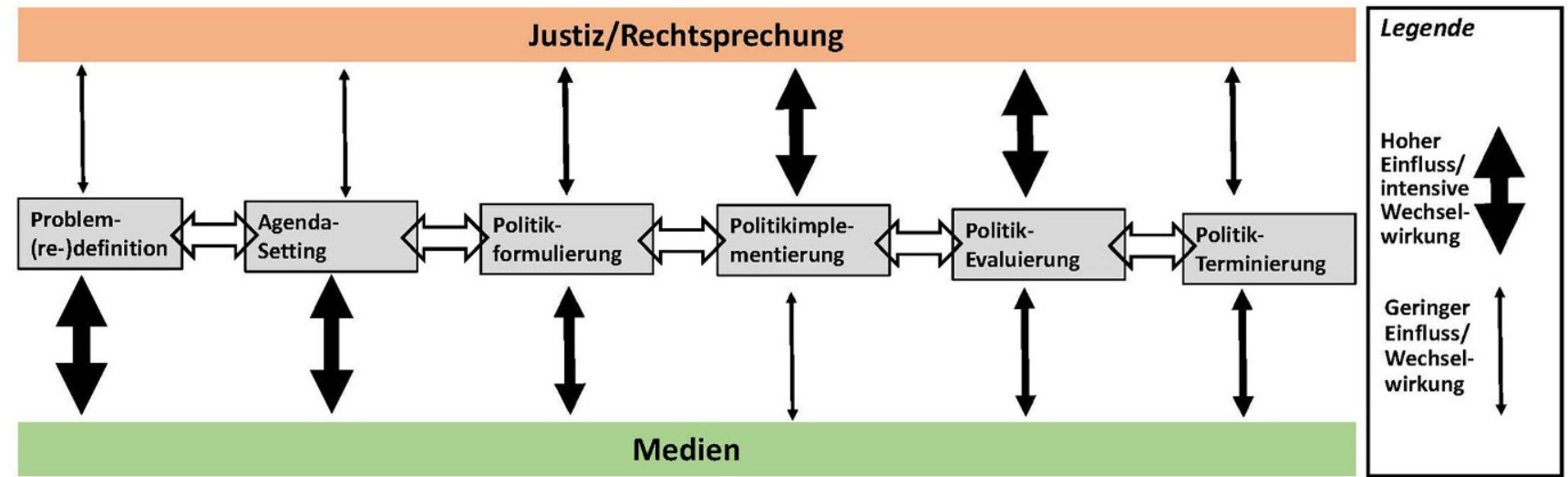

Abbildung 1 Interdependenzen von Medien und Justiz/Rechtsprechung zur Politik in den Phasen des Policy-Cycle (insbesondere Hypothesen 3, 4, 5). (Quelle: Eigene Darstellung in Anlehnung an Fawzi 2014a und Fawzi 2014b)

\subsubsection{Die abnehmende Bedeutung des Medieneinflusses im Verlauf des Policy-Cycle}

Fawzi (2014a: 438) formuliert als zentrale These, dass der Einfluss der Medien in der Problemdefinitionsphase besonders hoch ist, um dann in den nachfolgenden Phasen schrittweise abzunehmen. Da jedoch nur wenige Studien systematisch vergleichend den Einfluss der Medien auf Politik in allen Phasen des Politikprozesses (policy cycle) untersuchen, kann diese These vor allem aus der Zusammensetzung der Fülle von Einzeluntersuchungen aus einzelnen Phasen abgeleitet werden. Für die einzelnen Phasen lässt sich Folgendes festhalten:

Problemdefinition und Agenda-Setting: Während es sich bei der Phase der Problemdefinition vor allem um einen gesellschaftlichen Prozess unter Beteiligung der Politik handelt, in dem es darum geht, öffentliche Aufmerksamkeit zu erzeugen oder zu begrenzen, ist das Agenda-Setting, also die Setzung der durch die Politik zu bearbeitenden Probleme, bereits ein primär politischer Prozess. Dieser wird zwar meist von gesellschaftlichen Gruppen maßgeblich mitgestaltet, kann aber gleichwohl in Teilen jenseits der medialen Aufmerksamkeit stattfinden (Blum/Schubert 2011: 108). Nicht selten sind es externe Schocks und Krisen, die die Themendefinition auslösen (Jann/Wegrich 2014: 108f.). Zudem gibt es auch in der Raumplanung wiederkehrende Konjunkturen von Themen (vgl. Downs 1972; RußMohl 1993; Gravert/Günzel/Volkmann et al. 2013: 28). Ob ein Thema innerhalb eines Zeitfensters (opportunity window) den Schritt von der Problemdefinition auf die politische Agenda schafft, hängt auch davon ab, ob die politische Sichtweise mit der öffentlichen Meinung übereinstimmt und eine politische Lösung für das Problem nahelegt (Kingdon 1995: 146ff.; Mehta 2011: 35 f.). Das Problem muss in den Medien als relevant und lösbar dargestellt werden und es bedarf politischer Unternehmer, die sich dieses Problems annehmen und dabei Kontakt zu den
Medien suchen, weil sie diese als Indikator für Stimmungswechsel der Bevölkerung ansehen (Maurer 2013: 14). Medien haben politischen Einfluss weniger dadurch, was sie $\mathrm{zu}$ bestimmten Themen vermitteln, sondern dadurch, welche Themen sie überhaupt aufgreifen. Sie bestimmen nicht, was die Bevölkerung über etwas denkt, sondern worüber sie nachdenkt (Matthes 2007: 93). Als mögliche Wirkungsmodelle des Einflusses von Medien auf die Politik in den ersten beiden Phasen des Politikzyklus werden Problemthematisierung (Agenda-Setting), Rahmenbedingungen bzw. Versuchsanordnung (Framing), Zuschreibung (Priming) oder die Schweigespirale ${ }^{5}$ formuliert.

Im Agenda-Setting-Modell wird den Medien die Möglichkeit zugestanden, verschiedene Sichtweisen eines Themas vorzugeben, die die Selektionsmöglichkeit für weitere Informationen von vornherein verengen (Schulz 2009: $109 \mathrm{ff}$.). Das Framing als „Second-Level-Agenda-Setting“, das die Themen in einen bestimmten Rahmen setzt und auf den Mechanismen Selektion und Betonung (Salienz) basiert, ist hierbei zentral. Welche Sichtweise letztlich die Deutungshoheit erlangt, hängt auch davon ab, inwieweit die Medien das Framing strategischer Kommunikatoren aus der Politik übernehmen (Matthes 2007: $17 \mathrm{ff}$.). Um ein Thema so erfolgreich in der Öffentlichkeit zu platzieren, dass es den Scheitelpunkt (tipping point) der Aufmerksamkeit erreicht, bedarf es meist einer Storyline (Handlung), eines stimmigen ,roten Fadens' (Schwänke 2005). Der Begriff Priming bezieht sich darauf, welche positiven oder negativen Wirkungen die gesetzten Themen für bestimmte Politiker haben, weil sie in bestimmter Weise mit diesem Thema verbunden werden. In diesem Prozess erfolgt auch die Zuschreibung von politischer Verantwortung (Gerhards/ Offerhaus/Roose 2009: 545). Mit dem Begriff der Schwei-

\footnotetext{
${ }_{5}^{5}$ Politik und Medien verschweigen dabei wechselseitig bestimmte Themen, weil sie von deren Thematisireung weder politischen (Politik) noch kommerziellen (Medien) Nutzen haben.
} 
gespirale ist schließlich die gewisse Konkordanz der Medien im Agenda-Setting zur Politik angesprochen, die dazu führt, dass bestimmte Themen dauerhaft gar nicht auf die Agenda geraten (Schulz 2009: 106).

Empirische Untersuchungen gehen der Frage des Einflusses der Medien auf das Agenda-Setting häufig durch den Abgleich von Medienagenden und politischen Agenden nach. Nach der Medialisierungsthese müssten die politischen Agenden den Medienagenden folgen; die Befunde sind allerdings nicht eindeutig. Medienagenda und politische Agenda sind oft gar nicht kongruent zueinander (Kleinen-von Königslöw/Meyer/Vonbun et al. 2014). Wenn sie es aber sind, kann die Behandlung eines Themas in den politischen Entscheidungsprozessen zeitlich der öffentlichen Debatte folgen oder aber auch vorausgehen (Cobb/Ross/Ross 1976). Was die Form des Einflusses der Medien betrifft, so werden drei Wirkungsmechanismen unterschieden: Erstens kann der direkte Einfluss durch persuasive und kognitive Wirkungen auf Politiker erfolgen. Zweitens kann die Politik antizipativ auf die durch Medien vermutlich beeinflusste Meinung der Bevölkerung reagieren (Kepplinger 2007; Reinemann 2010: 284). Drittens kann die Politik auf die öffentliche Agenda reagieren, die vorher tatsächlich durch Medien beeinflusst wurde (Reinemann 2010: 281; Maurer 2013: 134; Fawzi 2014b: 101). Entscheidend ist letztlich nicht, ob die Medien die öffentliche Meinung beeinflussen, sondern dass die Politik annimmt, sie würden das tun und entsprechend handelt, weil sie sonst Gefahr läuft, bei Wählern als nicht responsiv oder gar inkompetent wahrgenommen zu werden (Walgrave/Van Aelst 2006: $99 \mathrm{ff}$.; Maurer 2013: 11, 22f., 176, 223; Hufnagel 2014: $270 \mathrm{ff}$.).

In der Phase der Politikformulierung werden aus artikulierten Problemen, Vorschlägen und Forderungen auf den Agenden konkretere staatliche Programme, wobei die Diskussion von Handlungsalternativen zentral ist (Jann/ Wegrich 2014: 111). Auch diese Phase gilt mittlerweile als offener gesellschaftlicher Prozess. Untersuchungen etwa zur deutschen Gesundheitspolitik belegen allerdings, dass Medien auch in der Politikformulierungsphase zumindest insofern einen Einfluss hatten, als die Entscheidungen beschleunigt und (in diesem Fall) in ihrer Qualität verschlechtert wurden (Fawzi 2014a: 451). Intensive Berichterstattung beeinflusst in jedem Fall das Verhandlungsklima, die Dauer und die Ergebnisqualität. Eine größere Rolle mögen auch gezielte Indiskretionen von Verhandlungsteilnehmern einnehmen, die den Medien Informationen zuspielen. Gleichwohl erscheint die Rolle der Medien in dieser Phase insgesamt deutlich geringer als in der vorangegangenen Phase. Die vorliegenden Untersuchungen (insbesondere Fawzi 2014b) bestätigen die Vermutung, dass die Beziehung zwischen Medienagenda und politischen Maßnahmen nur am Anfang der Themenkarriere eng ist, zum Zeitpunkt der Politikformulierung bereits nicht mehr. Nicht selten werden Beschlüsse sogar erst gefasst, wenn sie von den Medienagenden wieder verschwunden sind und dann medial unbeachtet bleiben (Maurer 2013: 33). In eine ähnliche Richtung geht die Beobachtung, dass manche Beschlüsse $\mathrm{zu}$ bewusst medial ungeeigneten Zeiten (z. B. während konkurrierender Ereignisse etwa aus dem Sport, zu Tagesrandzeiten, nach einer Phase des ,Aussitzens') gefasst werden.

Eine Inhaltsanalyse von drei bundesdeutschen Haushaltsdebatten aus mehreren Legislaturperioden, also aus der Phase der Politikformulierung, zeigte, dass die Redner sich in ihren Referenzen nicht stärker auf Medien bezogen als auf Bürger und andere Politiker. Auch war im Zeitverlauf kein Bedeutungsgewinn der Medien als Referenz zu beobachten, eher gewannen Experten als explizite Referenz an Bedeutung (Vowe/Dohle 2008: $11 \mathrm{ff}$.). Untersuchungen in den USA und Dänemark ergaben, dass Budgetausgaben von der Medienberichterstattung unbeeinflusst blieben (Fawzi 2014a: 451). Der sogenannte CNNEffekt schließlich, der den zeitlichen unmittelbaren Einfluss der Berichterstattung der Medien auf konkrete auslandspolitische Entscheidungen beschreibt (Löffelholz 2017), gilt für das politische Alltagsgeschäft nur selten (Fawzi 2014a: 451). Die in der Phase der Politikformulierung dominierende Verhandlungslogik scheint zur Medienlogik nur bedingt kompatibel. Gleichwohl haben Medien alleine durch ihre bloße Existenz hier in gewisser Weise zumindest einen indirekten Einfuss: Ein Großteil der Aushandlungsprozesse im Vorfeld der Entscheidungen wird nämlich von der Politik der direkten Öffentlichkeit bewusst entzogen, der punktuelle Medienausschluss gehört zu den Funktionsprinzipien der Politik (Fawzi 2014a: 443).

Implementation, Evaluierung, Terminierung: Die Implementation beinhaltet die Programmkonkretisierung sowie die Ressourcenbereitstellung zur Durchführung der Programme. In der Evaluationsphase geht es grundsätzlich um die rückblickende oder parallel zu den Prozessen verlaufende Bewertung der umgesetzten Politik. Für diese Phase liegen deutlich weniger bzw. überhaupt keine expliziten Forschungsbefunde vor, was den Einfluss der Medien angeht (Fawzi 2014a: 453 ff.). Expertenbefragungen lassen vermuten, dass auch für diese Phase zwar ein Medieneinfluss angenommen werden kann, der aber nur im Einzelfall bedeutend ist (Fawzi 2014a: $453 \mathrm{ff}$.).

Aufgrund der dargelegten Plausibilität der Hypothese, dass der Medieneinfluss mit zunehmendem Prozessfortschritt im Policy-Cycle abnimmt, nimmt auch die Intensität der Forschungen hierzu mit jeder Phase ab. Dadurch wird die Bedeutung der Medien in den ersten Phasen im Vergleich zu den anderen vermutlich stärker eingeschätzt, als sie real ist (Fawzi 2014a: $457 \mathrm{ff}$.). 


\subsubsection{Von der Medialisierungsthese zur Analyse von Wechselwirkungen}

Die vorangegangenen Ausführungen machen deutlich, dass Medien diskursive Macht haben. Durch ihre Berichterstattung können sie politische Entscheidungen maßgeblich beeinflussen. Nur selten - etwa beim investigativen Journalismus (vgl. Waldherr 2008) - fungieren sie dabei jedoch als aktive Agenda-Setter, die durchgängig eigene Positionen vertreten. Sie dienen vielmehr häufig als ,Aufmerksamkeitsscheinwerfer' für Themen und als Lieferanten für Interpretationen. Sie konstruieren durch die Selektion kontingenter Probleme eine öffentliche Agenda (Voltmer 1998: 32) und können damit Problemlösungskorridore verengen, bevor der institutionelle Entscheidungsprozess in Gang gekommen ist („Gatekeeper-Bias“) (Wolling 1999: 228; Burkart 2003: 7; Maurer 2013: 14f., 32; Walthert 2013: 13). Den negativen Einschätzungen dieser Bedeutung der Medien - die Trivialisierung der Kommunikation, die Entsachlichung und Personalisierung, die Fragmentierung der Öffentlichkeit und die bloße Anpassung der Politik an die Medienlogik, der Autonomieverlust und die Entauthentisierung der Politik (Schulz 2009: 105) - stehen auch optimistische Bewertungen gegenüber: die Konstitution einer politischen Öffentlichkeit, die Politikvermittlung durch Kommunikation und die Kontrolle des politischen Machtmissbrauchs (Marcinkowski/Steiner 2010: 66, 73). Medien können Lernprozesse bewirken und so politische Blockaden in Institutionen lösen. Sie verhelfen dem politischen System zu mehr Responsivität und Effektivität und schaffen somit Legitimität (Voltmer 1998: 31 ff.; Vetter 2008: 17; Schulz 2009: 114; Maurer 2013: 13). Umfassende Analysen der Hauptnachrichtenberichterstattung im deutschen Fernsehen kommen beispielsweise zu dem Ergebnis, dass auch in den privaten Sendern nicht von einer Entpolitisierung gesprochen werden kann und dass das Meinungsspektrum der Parteien - in den öffentlichen noch etwas mehr als in den privaten Sendern - durchaus angemessen vertreten ist (Kempf 2007).

Es wäre überzogen, generell von einer zunehmenden und einseitigen Bestimmung der Politik und der politischen Vertreter durch die Medien oder einzelne Medienvertreter und gleichzeitig von einer Entpolitisierung der Medien und damit von einer Entpolitisierung der Politik durch die Medien zu sprechen. Unterschiedliche Argumente stehen dem entgegen (vgl. Classen/Arnold 2010: 15). Viele Politikfelder stehen überhaupt nicht im medialen Fokus (Marcinkowski/ Steiner 2010: 65). Der Medieneinfluss bezieht sich vermutlich vor allem auf symbolische Politik in den ersten Phasen des Policy Cycle und weniger auf Entscheidungspolitik (Maurer 2013: 26). Die Politik hat Gegenmechanismen entwickelt: Einerseits werden Teile des Politikgeschehens überhaupt nicht mehr öffentlich kommuniziert, andererseits wird mediale Aufmerksamkeit von der Politik offensiv ge- nutzt - bis hin zum ,Spinning ' als gezielter Beeinflussung der Medien (Baumgartner/Jones 2009: 43; Green-Pedersen/ Stubager 2010: 669; Marcinkowski/Steiner 2010: 52; Reinemann 2010: 289). Nicht von ungefähr wird seitens der Medien häufig die bloße Duplizierung der Regierungserklärungen kritisiert (Wagner 2007; Ridder 2014). Die Medien müssen ihre Kommunikationslogik an die Politik anpassen, was diese in unterschiedlichem Maße tun: Tageszeitungen und Fernsehnachrichten entsprechen eher der medialen Kommunikationslogik, Talkshows und Politikerreden eher der politischen Kommunikationslogik (Maurer 2009: 169f.). Ein wichtiger Teil der Medien unterliegt nach wie vor formaler politischer Kontrolle, auch wenn durch die politisch gewollte - Liberalisierung der Medienlandschaft sich der direkte institutionelle Einfluss der Politik auf die Medien gelockert hat (Mai 2008). Insgesamt noch wichtiger ist, dass auch die Medien einem ,Herdentrieb“ der Aufmerksamkeit unter gestiegenem wirtschaftlichem Druck folgen, der eine bewusste politische Beeinflussung vermutlich sogar erschwert (Heye 2014: 48f.; Reinemann/Baugut 2014: 486).

Aus diesen Gründen hat sich die Medienforschung zum Teil von der These des einseitigen Einflusses der Medien abgewandt und widmet sich eher der Herausarbeitung der Mechanismen des wechselseitigen Einflusses von Medien und Politik (Classen/Arnold 2010: 18) und den im Zusammenhang damit geführten Interaktionen zwischen Politikern und Medienvertretern innerhalb von Akteurnetzwerken (Maurer 2013: 31). Medienvertreter, insbesondere „Alpha-Journalisten" (Krüger 2013), sind intensiv mit politischen Akteuren verflochten, vor allem mit jenen, die auf den Fortgang des Geschehens den vermutlich größten Einfluss haben. Diese Netzwerke sind aufgrund bestehender geistiger Berührungspunkte zwischen Politikern und Journalisten überhaupt erst entstanden. Die Medien versorgen die Politik mit Aufmerksamkeit und erhalten im Gegenzug Informationen, die sich ökonomisch nutzen lassen. Die Medien haben dabei als Zwischenhändler von Aufmerksamkeit katalytische Wirkung, greifen aber nicht in die sonstige politische Leistungserstellung ein (Kabalak/Priddat/Rhomberg 2008).

Diese Ergebnisse zu den wechselseitigen Einflüssen von Akteuren aus Politik und Medien können vor dem Hintergrund der jeweiligen institutionellen Kontexte betrachtet werden. Vor allem durch international vergleichende Studien konnte herausgearbeitet werden, dass die Interdependenzen zwischen Medien und Politik auch vom institutionellen Kontext beeinflusst werden (Adam 2008; Maurer 2013: 10). Es können Präsidialsysteme von Konsensdemokratien mit Mehrparteiensystemen unterschieden werden (Hallin/Mancini 2004: 51, 61). In klientelistisch geprägten politischen Kulturen werden auch die Medien eher in solche klientelistischen Relationen eingebunden, zumal wenn sie selbst wenig professionalisiert sind (Maurer 2008: 84). 
Wie Marschall (2009) im internationalen Vergleich mehrerer Parlamente zeigt, passen diese ihre Medienstrategien an den Wandel der Medienlandschaft an, behalten aber gleichwohl eine für sie spezifische Medienpolitik bei. Auch innerhalb eines Landes muss zwischen den politischen Arenen, die unterschiedliche institutionelle Kontexte haben, differenziert werden. Beispielsweise wird in den USA die Themenagenda von Präsidenten mehr durch Medien beeinflusst als die der Parlamente (Maurer 2013: 32).

\subsection{Die Rolle der Justiz}

Die Frage des Verhältnisses von Justiz auf der einen und Politik und Verwaltung auf der anderen Seite ist in der juristischen und politikwissenschaftlichen Debatte europaweit und in den USA (z. B. Landfried 1984; Hönnige 2007; Fornauf 2010; Jeschke 2010; Albrecht 2014) schon seit Längerem ein Thema. Die Gerichte müssen das Problem auslegungsbedürftiger bis gar widersprüchlicher Rechtsgrundlagen (incomplete contracting) durch Urteile lösen und fungieren dabei nicht selten als Schlichter in Konflikten (Hwang 2011: 323; Höreth 2013: 52). Aus juristischer Sicht geht mit der auf die Dynamisierung aller Lebensbereiche in allen Rechtsbereichen zurückzuführenden Zunahme ,unbestimmter Rechtsbegriffe ‘ zunächst eine Kompetenzverschiebung von der Rechtsetzung zur ausführenden Verwaltung und Politik einher, die dann unter Umständen durch die Rechtsprechung wieder ,eingefangen' werden muss. Zumindest gegenüber der Verwaltung sind nach der Gewaltengliederung des Grundgesetzes die Gerichte sogar übergeordnet (Pieroth/Hartmann 2008: 464; Projektgruppe „Richterliche Selbstverwaltung“ 2011: 122), worin das dem Rechtstaat immanente grundsätzliche Misstrauen gegenüber einer ,selbstherrlichen“ Verwaltung zum Ausdruck kommt (Mehde 2010: 379).

Auch die Politikwissenschaft misst schon seit Längerem der Judikative eine eigene Rolle im Politikgestaltungsprozess bei (Hönnige/Gschwend 2010; Knill/Tosun 2015: 83 f.). Ihr Einfluss wird auf zweierlei Weise ausgeübt: entweder direkt durch die korrigierende Revision oder durch vorlaufende Selbstbegrenzung der Politik, wenn diese - im Hinblick auf mögliche Rechtsurteile - selbst Korrekturen ihrer Agenda vornimmt (Stone Sweet 2007). Die korrigierende Revision hat in der Regel einen Rückfall des PolicyCycle-Prozesses bis zur Problemredefinition und Agendagestaltung zur Folge und kann gar zu einer ergebnislosen Terminierung der Politik führen (Knill/Tosun 2015: 83f.). Zwei Verfahrenstypen sind dabei zentral und lassen sich im Policy Cycle nach ihren Zeitpunkten unterscheiden: Abstrakte Normenkontrollverfahren, die in den meisten europäischen Ländern und den USA eine wichtige Rolle spielen (Vanberg 1998; Herr 2003), finden in der Phase der Gesetzgebung, also der Politikformulierung, statt. Verfahrensge- genstand ist in der Regel ein bereits beschlossenes Gesetz. Lediglich bei der präventiven Normenkontrolle wird der Gesetzentwurf vor dem Beschluss geprüft (Bednarik 2012: 317 ff.). Konkrete Normenkontrollverfahren finden dagegen in der Implementationsphase (wenn z. B. ein Planungsgesetz aufgrund einer Klage gegen einen aus diesem Gesetz abgeleiteten Plan überprüft wird) oder gar erst in der Evaluationsphase (z. B. im Rahmen einer inzidenten Kontrolle eines rechtsverbindlichen Plans, auf dessen Grundlage z.B. eine Baugenehmigung verwehrt wird) statt. Prinzipale Kontrolle prüft dabei die unmittelbare Wirksamkeit einer Norm, inzidente Kontrolle die Tauglichkeit zur Lösung der Norm für ein nachgelagertes Problem (Merkel 2012: 31).

Am intensivsten wurde die Frage des Verhältnisses von Justiz und Politik für die Verfassungsgerichte diskutiert, hier liegen auch einige empirische und zum Teil vergleichende Forschungsarbeiten vor. Juristen betonen tendenziell eher die traditionelle reaktive Rolle der Gerichte, viele Politikwissenschaftler messen ihnen jedoch eine aktivere Rolle bei (Rühmann 2014: 38). Wie hoch ihre Bedeutung ist und wie ihre Rolle genau ausfällt, dazu gibt es unterschiedliche Ansichten. Überholt sein dürfte die Haltung, die die Verfassungsgerichte nur als ,,verlängerten Arm der parlamentarischen Opposition“ versteht (Stüwe 2001; vgl. auch Landfried 1984: 176). Eine andere und plausiblere Position sieht dagegen Gerichte als Vetospieler. Damit hätten die Gerichte - namentlich das Verfassungsgericht - eine so zentrale Rolle, dass sie sogar als differenzierendes Kernmerkmal politischer Systeme und ihrer Reformfähigkeit gelten könnten (Tsebelis 2002). Zu einer wiederum anderen Einschätzung, die weniger die negativ-reaktive Rolle der Gerichte, sondern ihre positive gestaltende Funktion betont, kommt Hönnige (2007) in seinem empirischen Vergleich von Urteilen des deutschen und des französischen Verfassungsgerichts: Unabhängig vom institutionellen Kontext der zwischen diesen beiden Ländern im europäischen Vergleich maximal variiert - treten beide Verfassungsgerichte quasi als eine dritte Parlamentskammer neben Regierung und Opposition nicht nur als Kontrolleure und Vetospieler, sondern auch als Policy-Seeker ${ }^{6}$ und strategischer Akteur auf, der eigene Ziele verfolgt. Auch wenn die Gerichte formal über keine Gesetzgebungsinitiative verfügen, wirken sie so zum Teil als aktiver Agenda Setter und gehen damit über die Rolle des reinen Vetospielers hinaus. Dabei sind die legalen Motive in der richterlichen Argumentation nicht die einzigen Motive; politische Einstellungen und Reputationsfaktoren kommen hinzu. Dieses Zusammenspiel von Regierung, Opposition und Gerichten hat durchaus Vorteile für die Gesetzgebung: Vanberg (1998) kommt aufgrund spieltheoretischer Modellierungen zumindest für die USA zu dem Ergebnis, dass abstrakte Normenkontrollverfahren in

\footnotetext{
${ }^{6}$ Ein Akteur, der sogar eigene Politikziele formuliert.
} 
Prozessen der Gesetzgebung ein effizientes Instrument der Konsensbildung zwischen Regierung und Opposition darstellen, vorausgesetzt die Auffassungen der Gerichte weichen nicht zu stark von denen der Regierung ab.

Die Verfassungsgerichte haben sich demnach vom kontrollierenden Rechtsetzer zum Teil sogar zum positiven Rechtsetzer entwickelt, dessen Positionen von der Politik nicht selten antizipiert werden. Diese Beobachtung wird bis hin zur Behauptung einer ,dritten Parlamentskammer“ oder gar einer „Nebenregierung“ pointiert (Hönnige/ Gschwend 2010: 508), was noch zutreffender ist, wenn die eigentlichen legislativen politischen Organe, wie auf der Ebene der EU, nur geringe Steuerungskompetenz haben (Höreth 2013: 69f.). Stone Sweet (2000: 112) geht daher sogar bis zur These, die Parlamentssouveränität in Europa sei durch die Souveränität der Verfassungsgerichte ersetzt. Gegen diese Zuspitzung sprechen allerdings in erster Linie - zumindest für Deutschland - die Quantitäten. Bis 2007 wurden gerade einmal 2,5\% der Gesetzesentwürfe vor dem Bundesverfassungsgericht beklagt, pro Jahr landeten drei abstrakte und 33 konkrete Normenkontrollverfahren vor dem Bundesverfassungsgericht. Insgesamt hat das Bundesverfassungsgericht bislang lediglich 5\% aller vom Bundestag und Bundesrat verabschiedeten Gesetze beanstandet, wobei kein Trend nach unten oder oben erkennbar ist (Hönnige/Gschwend 2010: 510f.). Die Gerichte nehmen die Rolle des Letztentscheiders nicht durchgängig wahr. Ob sie als „,bedingter Vetospieler“ (Rühmann 2014: 84) fungieren, hängt von ihren strategischen Überlegungen und vom politischen System (Handlungsmöglichkeiten der Opposition) und den politischen Konstellationen ab. Wenn in Phasen einer Großen Koalition die Opposition zu klein ist, um das Gericht anzurufen, ist dessen Rolle geringer (Rühmann 2014).

Die wichtigste Rolle des Verfassungsgerichtes lässt sich weniger als die des Sprechers des „letzten Wortes“, sondern eher als die eines Wettbewerbshüters der Politik (Petersen 2014) oder eines Wegweisers im politischen Prozess bezeichnen (Lang 2014: 32). Die faktische Wirkung der Verwaltungsgerichtsbarkeit bleibt die einer "fleet in being“ (Mehde 2010: 383), die zwar nicht ständig aktiv ist, aber bei Bedarf eingeschaltet werden kann und insofern eine flächendeckende antizipierende Kontrollwirkung hat. Auch wenn Gerichte bisweilen als Vetospieler, als gleichberechtigter Akteur neben Regierung und Opposition und sogar als Politikgestalter fungieren können, bleibt dennoch die Gefahr ihrer einseitigen Machtausübung im System von Kontrolle und Überwachung (checks and balances) insofern insgesamt limitiert, als Gerichte von sich selbst aus nicht aktiv werden können, um eine eigene machtpolitische Agenda dauerhaft zu verfolgen, sondern angerufen werden müssen (Höreth 2013: 49). Demokratietheoretisch ist die starke Stellung der Gerichte insofern unproblematisch, als sie auch die Rolle des Minderheitenschutzes wahrnehmen (Drossel 2014). Sie agieren zudem nicht voraussetzungslos: Die Richter des Bundesverfassungsgerichts sind von den politischen Gremien gewählt und stehen unter politischer Einflussnahme sowie im Fokus der Öffentlichkeit, der Medien und anderer Gerichte. Justitialisierung der Politik bedeutet also keine generell zunehmende Dominanz der Justiz über die Politik, sondern ihre wechselseitige Durchdringung. Politik wird zunehmend in dem Spannungsdreieck zwischen Regierung, Opposition und Gerichten gestaltet (Stone Sweet 2007: 74). Dabei wird die Bedeutung informaler Kooperation in der Rechtsprechung abseits der formalisierten Arenen zwar auch in den Rechtswissenschaften zunehmend akzeptiert (Croon 2014), ist jedoch kaum erforscht.

\section{Fazit und Hypothesen für die weitere Forschung}

Die Auswertung der internationalen und deutschsprachigen Literatur machte zunächst deutlich, dass die Rollen der Medien und der Rechtsprechung in Planungsprozessen kaum erforscht ist. Dagegen wurde in anderen Politkfeldern (z. B. Außenpolitik, Finanzpolitik) zumindest die Frage des Verhältnisses von Medien und Politik intensiver beleuchtet. Nur rudimentär wurde allerdings auch hier das Zusammenspiel von Justiz und Politik betrachtet.

Die Thesen einer Medialisierung und Judizialisierung, also den Vermutungen einer generellen Zunahme des Einflusses der Dritten und Vierten auf die Erste und Zweite Gewalt, haben sich eher zur These eines intensivierten Wechselverhältnisses von Medien und Justiz einerseits und Politik andererseits entwickelt, in denen eher mittelbare Einflussmechanismen zentral sind. Für das Thema des Medieneinflusses sind diese Wirkmechanismen besser erforscht: Der wichtigste Einfluss dürfte in der vorsorgenden Vorwegnahme eines vermuteten Einflusses der Medien auf die Politik durch die Politik selbst liegen. Dass die Politikprozesse von den medialen Mechanismen zunehmend durchdrungen werden, heißt jedoch nicht, dass einzelne institutionalisierte Medien konkrete politische Entscheidungen verstärkt bestimmen. Vielmehr unterliegen auch Medien zunehmend Zwängen wie etwa dem zur Ökonomisierung der Berichterstattung. Und die Politik verfügt durchaus über Mechanismen, sich medialer Berichterstattung zu entziehen. Von daher hat sich die Forschung zur Frage des Medieneinflusses auf Politik zum Teil in Richtung der Analyse von Akteurnetzwerken zwischen Politik und Medienvertretern verändert. Die Interaktionen in diesen Netzwerken und der Verlauf sowie das Ergebnis bestimmter Entscheidungen dürften auch von institutionellen Hintergründen beeinflusst 
sein, die beispielsweise zwischen Ländern, Politikfeldern und Medientypen variieren.

Als abschließendes Ergebnis der Betrachtung hinsichtlich der Raumplanung lassen sich für empirische Forschungen folgende Hypothesen formulieren, die teilweise auch in Abbildung 1 ausgedrückt sind.

Hypothese 1 Sowohl zwischen Medien und Politik als auch zwischen Rechtsprechung und Politik bestehen nicht nur einseitige, sondern wechselseitige direkte und indirekte Abhängigkeiten.

Hypothese 2 Die Medien und noch mehr die Justiz haben im ,Normalfall der Planung' nur wenig Einfluss auf die Raumplanungsprozesse, da die meisten Fälle medial uninteressant sind und statistisch betrachtet nur in wenigen Planungsfällen Gerichte angerufen werden. In den wenigen Planungsfällen, in denen diese beiden Akteurgruppen involviert sind, dürfte ihr Einfluss jedoch sehr hoch bis mitentscheidend sein.

Hypothese 3 Der Policy-Cycle hat sich zu der Frage des Einflusses von Medien auf Politik als eine sehr brauchbare Untersuchungsheuristik erwiesen. Der Einfluss des Akteurs Medien (vgl. Kapitel 3.1) auf der einen und des Akteurs Justiz (vgl. Kapitel 3.2) auf der anderen Seite bzw. die Interdependenzen dieser beiden Akteurgruppen mit der Politik variieren systematisch nach den Phasen des PolicyCycle. Auch deswegen ist eine gemeinsame Betrachtung der Akteurgruppen Medien und Gerichte, die gemeinsam weite Teile der Politikprozessphasen beeinflussen können, erhellend.

Hypothese 4 Der Medieneinfluss nimmt mit den Stufen im Policy-Cycle zunächst ab (vgl. Kapitel 3.1): Die Medien haben vor allem in der Phase der Problemdefinition und - demgegenüber bereits etwas geringer - in der Phase des Agenda-Setting Einfluss auf die Politik bzw. hohe Wechselwirkungen mit der Politik. Das oben dargestellte internationale Beispiel (Oak Ridges Moraine; Edey/Seasons/ Whitelaw 2006) lässt die Vermutung zu, dass Planungsthemen häufig einen Zusatzimpuls benötigen, um überhaupt auf die Medienagenda zu geraten, etwa eine für die Öffentlichkeit interessante Verfehlung eines Politikers im Zusammenhang mit der Planung. In der Implementation, die sich in weiten Teilen als nicht öffentlicher Verwaltungsvollzug darstellt, sind diese Einflüsse nach den wenigen vorliegenden Studien aus anderen Politikfeldern jedoch geringer, in den Phasen der Politikformulierung moderat. Für die späteren Phasen liegen kaum Forschungsergebnisse vor. Hier könnten allerdings Untersuchungen für Raumplanungsthemen neue Forschungsimpulse setzen. Denn Konflikte mit der Öffentlichkeit werden oft erst in der Implementations- phase - im Extremfall der Bauphase - von Projekten deutlich. Hier können die Medien in quasi-evaluativer Funktion eine hohe Bedeutung als Verstärker und Investigatoren nicht funktionierender Implementation erhalten, wie vor allem die Debatten über Verzögerungen bei Großprojekten zeigen (z. B. Elbphilharmonie Hamburg, Flughafen BerlinBrandenburg). Aus diesem Grund wird in Abbildung 1 der Einfluss der Medien auf die Politik in den beiden letzten Phasen des Policy-Cycles wieder etwas stärker eingeschätzt als in der Phase der Implemetierung.

Hypothese 5 Die Justiz als Rechtsprechung - in Raumplanungsprozessen sind vor allem nachgeordnete Gerichte relevant - hat kaum Bedeutung in den ersten Phasen des Policy-Cycles. Sie erhält ihren Haupteinfluss erst in den Phasen der Implementierung von Gesetzen und vor allem ihrer Evaluierung, verstanden als rückblickende Bewertung. Wenn rechtliche Fragen in Raumplanungsprozessen eine Rolle spielen, ist sie die zentrale quasi-evaluierende Instanz (vgl. Kapitel 3.2).

Hypothese 6 Wenngleich Medien und Justiz/Rechtsprechung tendenziell in unterschiedlichen Phasen des Policy-Cycle Einfluss haben, so ist in bestimmten besonders öffentlichkeitswirksamen Raumplanungsprozessen auch die Frage des direkten Verhältnisses zwischen Medien und Gerichten von Interesse. Wie kritisch wird beispielsweise in Medien über ein Gerichtsurteil berichtet, passen sich Gerichte in ihrem Urteil einem medialen Erwartungsdruck nicht doch zumindest tendenziell an? Wenn die Rechtsprechung zum medialen Gegenstand wird, so ist sie vermutlich ähnlichen Mechanismen gegenüber den Medien ausgesetzt wie die Politik. Eventuell bilden sich auch hier entsprechende Netzwerke zwischen den Akteuren aus Justiz und Medien heraus (vgl. Kapitel 3.1).

Hypothese 7 Die Mechanismen der Ausübung des Einflusses auf die Politik unterscheiden sich zwischen Medien und Justiz etwa in der Art der Argumentation. Die Justiz trifft eher formal eingeengte Entscheidungen, während die Medien ihre Ansätze emotional variieren können. Jedoch gibt es auch Gemeinsamkeiten: Gerichte argumentieren nicht nur formaljuristisch und Medien nicht nur an Neuigkeitswerten orientiert. Herauszuarbeiten wären auch die spezifischen Rollen und Rollenverständnisse der Akteure von Medien und Justiz in den Planungsprozessen.

Hypothese 8 Institutionelle Kontexte und Akteurkonstellationen erklären den Einfluss der Medien und der Gerichte in Raumplanungsprozessen maßgeblich (vgl. Kapitel 3.2).

Diesen Hypothesen, die durch Fragen zu den Rahmenbedingungen, Akteuren, Planungsprozessen und Ergebnissen zu vertiefen wären, kann vor allem in national wie inter- 
national vergleichenden Analysen nachgegangen werden. So ist die Stellung der Medien und der Justiz in einer direkten Demokratie eine nuanciert andere als in der parlamentarischen Demokratie. Aber auch das Herausarbeiten von Gemeinsamkeiten und Unterschieden zwischen Themenfeldern der Raumplanung (z. B. Stadterneuerung vs. Raumordnung) könnte zu interessanten Forschungsergebnissen führen.

\section{Literatur}

Adam, S. (2008): Massenmedien als Herausforderer oder Agenturen nationaler Eliten? Eine Analyse der deutschen und französischen EU-Erweiterungsdebatte. In: Pfetsch, B.; Adam, S. (Hrsg.): Massenmedien als politische Akteure. Konzepte und Analysen. Wiesbaden, 116-143.

Albrecht, P.-A. (2014): Sicherungen für die Autonomie der Dritten Gewalt in Europa! In: Kritische Vierteljahresschrift für Gesetzgebung und Rechtswissenschaft 97, 4, 331-333.

Aleksandrov, E. (2015): News-Bias in der Medienberichterstattung über ,Stuttgart 21“? Eine Inhaltsanalyse der Berichterstattung der überregionalen Tagespresse. München.

Baringhorst, S. (2009): Politischer Protest im Netz - Möglichkeiten und Grenzen der Mobilisierung transnationaler Öffentlichkeit im Zeichen digitaler Kommunikation. In: Marcinkowski, F.; Pfetsch, B. (Hrsg.): Politik in der Mediendemokratie. Wiesbaden, 609634. $=$ Politische Vierteljahresschrift, Sonderheft 42.

Baumgarten, B.; Rucht, D. (2013): Die Protestierenden gegen „Stuttgart 21" - einzigartig oder typisch? In: Brettschneider, F.; Schuster, W. (Hrsg.): Stuttgart 21. Ein Großprojekt zwischen Protest und Akzeptanz. Wiesbaden, 97-126.

Baumgartner F. R.; Jones B. D. (2009): Agendas and Instability in American Politics. Chicago.

Becker-Ritterspach, L. (2015): Steuerung der Implementation von Flughäfen. Eine Untersuchung des Flughafenbaus in Frankfurt a.M. und Berlin-Brandenburg. Wiesbaden. doi: 10.1007/978-3658-07357-2

Bednarik, S. (2012): Präventive Normenkontrolle durch Verfassungsgerichte. Eine staatsrechtliche und rechtsvergleichende Untersuchung im europäischen Raum. Berlin.

Bernhard, U. (2012): Infotainment in der Zeitung. Der Einfluss unterhaltungsorientierter Gestaltungsmittel auf die Wahrnehmung und Verarbeitung politischer Informationen. Baden-Baden. = reihe Rezeptionsforschung 23.

Blum, S.; Schubert, K. (2011): Politikfeldanalyse. Wiesbaden.

Brettschneider, F.; Schuster, W. (2013): „Stuttgart 21“. Ein Großprojekt zwischen Protest und Akzeptanz. In: Brettschneider, F.; Schuster, W. (Hrsg.): Stuttgart 21. Ein Großprojekt zwischen Protest und Akzeptanz. Wiesbaden, 9-14.

Burkart, R. (2003): Medienwirkungsforschung - ein Einblick. In: Medienimpulse - Beträge zur Medienpädagogik 12, 46, 5-8.

Classen, C.; Arnold, K. (2010): Von der Politisierung der Medien zur Medialisierung des Politischen? Zum Verhältnis von Medien, Öffentlichkeiten und Politik im 20. Jahrhundert. In: Arnold, K.; Classen, C.; Kinnebrock, S.; Lerch, E.; Wagner, H.-U. (Hrsg.): Von der Politisierung der Medien zur Medialisierung des Politischen? Zum Verhältnis von Medien, Öffentlichkeit und Politik im 20. Jahrhundert. Leipzig, 11-28.

Cobb, R.; Ross, J.-K.; Ross. M. H. (1976): Agenda Building as a Comparative Political Process. In: The American Political Science Review 70, 1, 126-138.

Croon, J. (2014): Abseits der verfassungsrechtlichen Arenen - informale Kommunikation. In: Elser, D.; Eugster, A.; Kind, A.; Uffer, M.; Baumgartner, R.; Williner, K.; Schlegel, S.; Blonski, D.; Spring, A.; Grohsmann, I.; Häcki, R. (Hrsg.): Das letzte Wort -
Rechtsetzung und Rechtskontrolle in der Demokratie. Baden-Baden, 39-58.

Dohle, M.; Bernhard, U. (2014): Mediennutzung und -wahrnehmung von Bundestagsabgeordneten: Ersetzen oder ergänzen OnlineMedien klassische Informations- und Kommunikationskanäle? In: Zeitschrift für Parlamentsfragen 45, 4, 763-774.

Downs, A. (1972): Up and down with Ecology - The 'Issue Attention Cycle'. In: Public Interest 28, 38-50.

Drossel, J.-M. (2014): Das letzte Wort des Bundesverfassungsgerichts - ein undemokratischer Mechanismus? In: Elser, D.; Eugster, A.; Kind, A.; Uffer, M.; Baumgartner, R.; Williner, K.; Schlegel, S.; Blonski, D.; Spring, A.; Grohsmann, I.; Häcki, R. (Hrsg.): Das letzte Wort - Rechtsetzung und Rechtskontrolle in der Demokratie. Baden-Baden, 255-274.

Edey, R. C.; Seasons, M.; Whitelaw, G. (2006): The Media, Planning and the Oak Ridges Moraine. In: Planning, Practice and Research 21, 2, 147-161. doi: 10.1080/02697450600944632

Eibl, T. (2012): Die Schlichtungsgespräche zum Bahnprojekt „Stuttgart 21“. Entscheidungsfindung und Einfluss zwischen Deliberation und Agonismus. München.

Fawzi, N. (2014a): Chronisten, Agenda-Setter oder Politikmacher? Der Einfluss der Medien im politischen Prozess. In: Zeitschrift für Politik 61, 4, 437-460. doi: 10.5771/0044-3360-2014-4-437

Fawzi, N. (2014b): Machen Medien Politik? Medialisierung der Energiepolitik aus Sicht von politischen Akteuren und Journalisten. Baden-Baden.

Fischler, R. (2000): Communicative Planning Theory: A Foucauldian Assessment. In: Journal of Planning Education and Research 19, 4, 358-368. doi: 10.1177/0739456X0001900405

Fornauf, M. (2010): Die Unabhängigkeit der Dritten Gewalt im rechtsstaatlichen Strafrecht. Bedingungen fortschreitender Marginalisierung. In: Kritische Vierteljahresschrift für Gesetzgebung und Rechtswissenschaft 93, 3, 217-232.

Fürst, D. (2010): Akteure der Planung. In: Henckel, D.; von Kuczkowski, K.; Lau, P.; Pahl-Weber, E.; Stellmacher, F. (Hrsg.): Planen Bauen - Umwelt. Ein Handbuch. Wiesbaden, 18-21.

Gerhards, J.; Offerhaus, A.; Roose, J. (2009): Wer ist verantwortlich? Die Europäische Union, ihre Nationalstaaten und die massenmediale Attribution von Verantwortung für Erfolge und Misserfolge. In: Marcinkowski, F.; Pfetsch, B. (Hrsg.): Politik in der Mediendemokratie. Wiesbaden, 529-558. = Politische Vierteljahresschrift, Sonderheft 42.

Gravert, A.; Günzel, M.; Volkmann, A.; Wiechmann, T. (2013): Agenda-Setting in der Planung. Zur Karriere von stadtplanerischen Modethemen. In: RaumPlanung 167, 2, 27-32.

Green-Pedersen, C.; Stubager, R. (2010): The Political Conditionality of Mass Media Influence. When do Parties Follow Mass Media Attention? In: British Journal of Political Science 40, 3, 663-677. doi: 10.1017/S0007123410000037

Hallin, D. C.; Mancini, P. (2004): Comparing Media Systems. Three Models of Media and Politics. Cambridge.

Healey, P. (1996): The communicative turn in planning theory and its implications for spatial strategy formation. In: Environment and Planning B 23, 2, 217-234. doi: 10.1068/b230217

Herr, C. C. (2003): Die „neue“ Antragsbefugnis im Normenkontrollverfahren nach § 47 II VwGO. Baden-Baden.

Heye, U.-K. (2014): Medien und Politik, Nähe und Distanz - Interview mit Herlinde Koelbl. In: Stiftung Haus der Geschichte der Bundesrepublik Deutschland (Hrsg.): Unter Druck! Medien und Politik. Bielefeld, 32-49.

Hönnige, C. (2007): Verfassungsgericht, Regierung und Opposition. Die vergleichende Analyse eines Spannungsdreiecks. Wiesbaden.

Hönnige, C.; Gschwend, T. (2010): Das Bundesverfassungsgericht im politischen System der BRD - ein unbekanntes Wesen? In: Politische Vierteljahresschrift 51, 3, 507-530. doi: 10.1007/s11615010-0030-z

Höreth, M. (2013): Hemmungslos, aber ungefährlich? Der Gerichtshof der Europäischen Union als Verfassungsgericht im System 
der EU-Gewaltenteilung. In: Zeitschrift für Politik 60, 1, 48-71. doi: 10.5771/0044-3360-2013-1-48

Hufnagel, A. (2014): Der Einfluss der Medien auf Unternehmensentscheidungen. Wie Unternehmen die Medienberichterstattung beobachten, beurteilen und berücksichtigen. Baden-Baden. = Kommunikation in Politik und Wirtschaft 11.

Hwang, S.-P. (2011): Der „unbestimmte Rechtsbegriff“ als Rechtsbegriff. Zur normativen Kontrollfunktion der unbestimmten Rechtsbegriffe im technisch-wissenschaftlichen Zeitalter. In: Kritische Vierteljahresschrift für Gesetzgebung und Rechtswissenschaft 94, 4, 313-330.

In der Smitten, S. (2009): Online-Vergemeinschaftung. Potenziale politischen Handelns im Internet. Baden-Baden. = Internet-Research 31. doi: $10.5771 / 9783845214689$

Jann, W.; Wegrich, K. (2014): Phasenmodelle und Politikprozesse: Der Policy-Cycle. In: Schubert, K.; Bandelow, N. C. (Hrsg.): Lehrbuch der Politikfeldanalyse. München, 97-132.

Jarren, O.; Donges, P. (2011): Politische Kommunikation in der Mediengesellschaft. Eine Einführung. Wiesbaden.

Jeschke, M. (2010): Modelle einer selbstverwalteten Dritten Gewalt in Europa. In: Kritische Vierteljahresschrift für Gesetzgebung und Rechtswissenschaft 93, 3, 233-255.

Johnson, B. J.; Graves, M. (2011): Keeping it real. What planning can learn from reality TV. In: Journal of the American Planning Association 77, 3, 214-231. doi: 10.1080/01944363.2011.592128

Kabalak, A.; Priddat, B. P.; Rhomberg, M. (2008): Medien als Schnittstelle zwischen politischen und ökonomischen Strukturen - Politische Kommunikation in der Perspektive der Institutionenökonomie. In: Pfetsch, B.; Adam, S. (Hrsg.): Massenmedien als politische Akteure. Konzepten und Analysen. Wiesbaden, 52-72.

Keilbach, M. (2009): Rechtsschutz gegen Flächennutzungspläne. Hamburg.

Kempf, M. (2007): Tendenzen im TV. Die politischen Präferenzen der Hauptnachrichtensendungen. Saarbrücken.

Kepplinger, H. M. (2007): Politiker als Protagonisten der Medien. In: Zeitschrift für Politik 54, 3, 272-295. doi: 10.5771/0044-33602007-3-272

Kingdon, J. W. (1995): Agendas, Alternatives, and Public Policies. New York.

Kleinen-von Königslöw, K.; Meyer, T. M.; Vonbun, R.; Wagner, M.; Winkler, A. K. (2014): Die Sachthemen im Wahlkampf. In: Kritzinger, S.; Müller, W. C.; Schönbach, K. (Hrsg.): Die Nationalratswahl 2013. Wie Parteien, Medien und Wählerschaft zusammenwirken. Wien, 39-66.

Knill, C.; Tosun, J. (2015): Einführung in die Policy-Analyse. Opladen.

Krüger, U. (2013): Meinungsmacht. Der Einfluss von Eliten auf Leitmedien und Alpha-Journalisten - eine kritische Netzwerkanalyse. Köln.

Kupfer, D. (2014): Das Fachplanungsrecht in der neueren Rechtsprechung des Bundesverwaltungsgerichts, Fortschreibung 2014. In: Die Verwaltung. Zeitschrift für Verwaltungsrecht und Verwaltungswissenschaften 47, 1, 77-124.

Landfried, C. (1984): Bundesverfassungsgericht und Gesetzgeber: Wirkungen der Verfassungsrechtsprechung auf parlamentarische Willensbildung und soziale Realität. Baden-Baden.

Lang, A. (2014): Wider die Metapher vom letzten Wort: Verfassungsgerichte als Wegweiser. In: Elser, D.; Eugster, A.; Kind, A.; Uffer, M.; Baumgartner, R.; Williner, K.; Schlegel, S.; Blonski, D.; Spring, A.; Grohsmann, I.; Häcki, R. (Hrsg.): Das letzte Wort Rechtsetzung und Rechtskontrolle in der Demokratie. Baden-Baden, 15-38.

Lauth, H.-J.; Thiery, P. (2012): Politikfeldanalyse. In: Lauth, H.-J.; Wagner, C. (Hrsg.): Politikwissenschaft: Eine Einführung. Paderborn, 263-293.

Löffelholz, M. (2017): Kriegsberichterstattung in der Mediengesellschaft. In: Aus Politik und Zeitgeschichte 16/17, 25-31.

Mai, M. (2008): Macht und Gegenmacht: Zum Verhältnis politischer und medialer Macht. In: Jäckel, M.; Mai, M. (Hrsg.): Medien- macht und Gesellschaft. Zum Wandel öffentlicher Kommunikation. Frankfurt am Main/New York, 123-146.

Maier, T. (2013): „Stuttgart 21“ im Spiegel von Facebook-Aktivitäten der Befürworter und der Gegner des Projektes. In: Brettschneider, F.; Schuster, W. (Hrsg.): Stuttgart 21. Ein Großprojekt zwischen Projekt und Akzeptanz. Wiesbaden, 77-96.

Marcinkowski, F.; Steiner, A. (2010): Was heißt „Medialisierung“? Autonomiebeschränkung oder Ermöglichung von Politik durch Massenmedien? In: Arnold, K.; Classen, C.; Kinnebrock, S.; Lerch, E.; Wagner, H.-U. (Hrsg.): Von der Politisierung der Medien zur Medialisierung des Politischen? Zum Verhältnis von Medien, Öffentlichkeit und Politik im 20. Jahrhundert. Leipzig, 51-76.

Marschall, S. (2009): Medialisierung komplexer politischer Akteure - Indikatoren und Hypothesen am Beispiel von Parlamenten. In: Marcinkowski, F.; Pfetsch, B. (Hrsg.): Politik in der Mediendemokratie. Wiesbaden, 205-223. = Politische Vierteljahresschrift, Sonderheft 42.

Matthes, J. (2007): Framing-Effekte. Zum Einfluss der Politikberichterstattung auf die Einstellungen der Rezipienten. München. $=$ Reihe Rezeptionsforschung 13.

Maurer, M. (2009): Wissenvermittlung in der Mediendemokratie: Wie Medien und politische Akteure die Inhalte von Wahlprogrammen kommunizieren. In: Marcinkowski, F.; Pfetsch, B. (Hrsg.): Politik in der Mediendemokratie. Wiesbaden, 151-173. = Politische Vierteljahresschrift, Sonderheft 42.

Maurer, P. (2008): Einflussfaktoren auf die Handlungsautonomie der Medien im politischen Prozess westlicher Demokratien - Eine theoretische Analyse. In: Pfetsch, B.; Adam, S. (Hrsg.): Massenmedien als politische Akteure. Konzepte und Analysen. Wiesbaden, 73-91.

Maurer, P. (2013): Medieneinfluss aus der Sicht von Politikern und Journalisten. Ein deutsch-französischer Vergleich. Berlin. = Studien zur politischen Kommunikation 8.

Mehde, V. (2010): Verwaltungskontrolle als Daueraufgabe der Verwaltungsgerichtsbarkeit. In: Die Verwaltung. Zeitschrift für Verwaltungsrecht und Verwaltungswissenschaften 43, 3, 379-404.

Mehta, J. (2011): The Varied Roles of Ideas in Politics. From ,whether" to „how“. In: Béland, D.; Cox, R. H. (Hrsg.): Ideas and Politics in Social Science Research. Oxford, 23-46.

Mergel, T. (2010): Politisierte Medien und medialisierte Politik. Strukturelle Kopplungen zwischen zwei sozialen Systemen. In: Arnold, K.; Wagner, H.-U.; Classen, C.; Kinnebrock, S.; Lerch, E. (Hrsg.): Von der Politisierung der Medien zur Medialisierung des Politischen? Zum Verhältnis von Medien, Öffentlichkeit und Politik im 20. Jahrhundert. Leipzig, 29-50.

Merkel, M. (2012): Die Gerichtskontrolle der Abwägung im Bauplanungsrecht, insbesondere nach der Neuregelung der $\S \S 2$ III und 214 BauGB durch das EAG Bau. Berlin. = Schriften zum Öffentlichen Recht 1211.

Petersen, N. (2014): Verfassungsgerichte als Wettbewerbshüter des politischen Prozesses. In: Elser, D.; Eugster, A.; Kind, A.; Uffer, M.; Baumgartner, R.; Williner, K.; Schlegel, S.; Blonski, D.; Spring, A.; Grohsmann, I.; Häcki, R. (Hrsg.): Das letzte Wort - Rechtsetzung und Rechtskontrolle in der Demokratie. Baden-Baden, 5980.

Pfetsch, B.; Adam, S. (2008): Die Akteursperspektive in der politischen Kommunikationsforschung - Fragestellungen, Forschungsparadigmen und Problemlagen. In: Pfetsch, B.; Adam, S. (Hrsg.): Massenmedien als politische Akteure. Konzepte und Analysen. Wiesbaden, 9-26.

Pieroth, B.; Hartmann, B.J. (2008): Gewaltenübergreifende Bindungswirkung. Zur Maßgeblichkeit von Gerichtsentscheidungen für Behörden. In: Die Verwaltung 41, 4, 463-481. doi: 10.3790/ verw.41.4.463

Projektgruppe „Richterliche Selbstverwaltung“ (2011): Brandenburgische Erwägungen für eine Stärkung der Autonomie der Dritten 
Gewalt. In: Kritische Vierteljahresschrift für Gesetzgebung und Rechtsprechung 94, 2, 119-135.

Reinemann, C. (2010): Medialisierung ohne Ende? Zum Stand der Debatte um Medieneinflüsse auf die Politik. In: Zeitschrift für Politik 57, 3, 278-293. doi: 10.5771/0044-3360-2010-3-278

Reinemann, C.; Baugut, P. (2014): Alter Streit unter neuen Bedingungen. Einflüsse politischer Einstellungen von Journalisten auf ihre Arbeit. In: Zeitschrift für Politik 61, 4, 480-505. doi: 10.5771/0044-3360-2014-4-480

Richter, S. (2011): Zur Rolle von Öffentlichkeit und Medien in Planungsprozessen. In: RaumPlanung 156/157, 169-173.

Ridder, W. (2014): „Vierte Gewalt“ oder „Not-so-independent-broker“. Die Legende vom irakischen Atomwaffenprogramm und die Medien in den Vereinigten Staaten, dem Vereinigten Königreich und in Deutschland. In: Zeitschrift für Politik 61, 3, 263-283. doi: 10.5771/0044-3360-2014-3-263

Rühmann, F. (2014): Das Bundesverfassungsgericht als kaltgestellter Vetospieler? Möglichkeiten und Grenzen richterlicher Normenkontrolle unter den Bedingungen einer Großen Koalition. Norderstedt.

Ruß-Mohl, S. (1993): Konjunkturen und Zyklizität in der Politik: Themenkarrieren, Medienaufmerksamkeits-Zyklen und ,lange Wellen“. In: Héritier, A. (Hrsg.): Policy-Analyse. Kritik und Neuorientierung. Opladen, 356-368.

Schulz, W. (2009): Politischer Medieneinfluss: Metamorphosen des Wirkungskonzepts. In: Marcinkowski, F.; Pfetsch, B. (Hrsg.): Politik in der Mediendemokratie. Wiesbaden, 103-128. = Politische Vierteljahresschrift, Sonderheft 42.

Schwänke, U. (2005): Die Storyline-Methode. Donauwörth.

Selle, K. (2005): Planen. Steuern. Entwickeln. Der Beitrag öffentlicher Akteure zur räumlichen Entwicklung von Stadt und Land. Dortmund.

Stone Sweet, A. (2000): Governing with Judges. Constitutional Politics in Europe. Oxford.

Stone Sweet, A. (2007): The Politics of Constitutional Review in France and Europe. In: International Journal of Constitutional Law 5, 1, 69-92. doi: 10.1093/icon/mol041

Stuckenbrock, U. (2013): Das Projekt „Stuttgart 21“ im zeitlichen Überblick. In: Brettschneider, F.; Schuster, W. (Hrsg.): Stuttgart 21. Ein Großprojekt zwischen Protest und Akzeptanz. Wiesbaden, 15-76.

Stüwe, K. (2001): Das Bundesverfassungsgericht als verlängerter Arm der Opposition? Eine Bilanz seit 1951. In: Aus Politik und Zeitgeschichte 37/38, 34-44.
Sullivan, E. J. (2015): United States of America/Vereinigte Staaten von Amerika. In: Mitschang, S. (Hrsg.): Privatisation of planning powers and urban infrastructure. Frankfurt am Main, 153-166. = Berliner Schriften zur Stadt- und Regionalplanung 26.

Tsebelis, G. (2002): Veto Players. How Political Institutions Work. New York.

Vanberg, G. (1998): Abstract Judicial Review, Legislative Bargaining and Policy Compromise. In: Journal of Theoretical Politics 10, 3, 299-326. doi: 10.1177/0951692898010003005

Vetter, A. (2008): Lokale Bürgerbeteiligung: Ein wichtiges Thema mit offenen Fragen. In: Vetter, A. (Hrsg.): Erfolgsbedingungen lokaler Bürgerbeteiligung. Wiesbaden, 9-27.

Voltmer, K. (1998): Medienqualität und Demokratie. Eine empirische Analyse publizistischer Informations- und Orientierungsleistungen in der Wahlkampfkommunikation. Baden-Baden. = NomosUniversitätsschriften 94.

Vowe, G.; Dohle, M. (2007): Politische Kommunikation im Umbruch - neue Forschung zu Akteuren, Medieninhalten und Wirkungen. In: Politische Vierteljahresschrift 48, 2, 338-356.

Vowe, G.; Dohle, M. (2008): Welche Macht wird den Medien zugeschrieben? Das Verhältnis von Medien und Politik im Spiegel der Mediatisierungsdebatte. In: Jäckel, M.; Mai, M. (Hrsg.): Medienmacht und Gesellschaft. Zum Wandel öffentlicher Kommunikation. Frankfurt am Main/New York, 11-36.

Wagner, H. (2007): Vom Gespenst, das als „Vierte Gewalt“ erscheint. Bemerkungen zu einer Demokratiegefährdung, die sich als ihr Gegenteil ausgibt. In: Zeitschrift für Politik 54, 3, 324-351. doi: 10.5771/0044-3360-2007-3-324

Waldherr, A. (2008): Gatekeeper, Diskursproduzenten und AgendaSetter - Akteursrollen von Massenmedien in Innovationsprozessen. In: Pfetsch, B.; Adam, S. (Hrsg.): Massenmedien als politische Akteure. Konzepte und Analysen. Wiesbaden, 171-195.

Walgrave, S.; Van Aelst, P. (2006): The Contingency of the Mass Media's Political Agenda Setting Power: Toward a Preliminary Theory. In: Journal of Communication 56, 1, 88-109. doi: 10.1111/j.1460-2466.2006.00005.x

Walthert, L. (2013): Der Effekt von Massenmedien auf das Wahlverhalten. Ein Literaturüberblick. München.

Wolling, J. (1999): Politikverdrossenheit durch Massenmedien. Der Einfluß der Medien auf die Einstellungen der Bürger zur Politik. Opladen. 